\title{
À propos des suffixes appréciatifs de l'espagnol. Note de mimophonie
}

\author{
Samuel Bidaud* \\ Université de Bourgogne, Francia
}

On sait que Saussure avait posé dans le Cours de linguistique générale que le signe était arbitraire, c'est-à-dire qu'il n'y avait pas de lien entre son signifiant et son signifié. La preuve qu'il en donnait était essentiellement la suivante: la suite phonique chargée d'exprimer un sens varie d'une langue à l'autre. Ainsi n'y a-t-il aucun rapport entre la suite sonore boeuf en français et la suite sonore Ochs en allemand (Saussure, 1967:100). Cet argument permettait également à Saussure de rejeter l'idée selon laquelle les onomatopées étaient motivées, puisque, là encore, elles variaient d'une langue à l'autre.

Or, la question de l'arbitraire du signe avait été déjà discutée bien avant Saussure et l'est encore après lui, et l'on ne saurait considérer son point de vue à l'heure actuel comme fondé.

Déjà dans le Cratylede Platon, Cratyle soutenait que le son représentait la chose et que les mots étaient motivés, contrairement à ce que soutenait Hermogène, qui lui pensait, en pré-saussurien, qu'il n'y avait aucun rapport entre le signifié et le signifiant. L'âge classique a connu des débats similaires, et des grammairiens comme Court de Gébelin s'efforçaient de montrer que chaque lettre était douée d'une valeur sémantique en soi, par exemple les dentales, qui servent à nommer "les objets vastes et dominants", les "masses

\footnotetext{
* Para correspondencia, dirigirse a: Samuel Bidaud (samuel.bidaud@aliceadsl.fr), 1, rue Ernest Petit, 21000 Dijon, Côte d'Or, France/Francia.
} 
amoncelées", et tout ce qui protège, mais aussi, puisque "les dents sont aussi fermes que les lèvres sont mobiles et flexibles, les intonations qui en proviennent sont aussi fortes, aussi sonores, aussi bruyantes", et c'est par cette touche "qu'on tonne, qu'on retentit, qu'on étonne"(Court de Gébelin, 1776: 91-92). Le Président De Brosses allait également dans ce sens, et, grâce à une comparaison entre les différentes langues du monde, il montrait comment une même séquence phonique se retrouvait dans de nombreuses langues très différentes pour exprimer le nom de la mère et du père. $\mathrm{Au}$ vingtième siècle enfin, de nombreux linguistes se sont prononcés en faveur de la motivation du signe. C'est le cas de Jespersen, qui développe l'idée de phonosymbolisme, ou d'un autre grand linguiste danois, Louis Hjelmslev, qui écrit dans ses Principes de grammaire générale qu' "un des caractères fondamentaux du sujet parlant est sa grande naïveté: sans vouloir entendre raison, il croit fermement que les mots de sa langue maternelle sont tous

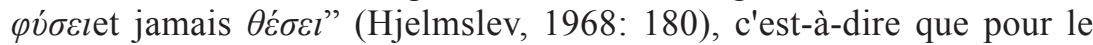
locuteur moyen, les mots de la langue sont motivés et ne pourraient pas se dire autrement. Hjelmslev souligne en outre que la forme peut influencer le sens. C'est également le point de vue de Jespersen, qui montre que certains mots peuvent même changer de sens à cause de leur phonétisme. C'est le cas d'un mot comme miniature, qui, sous l'influence du $i$, en vient à signifier l'idée de petitesse ( $i$ étant la voyelle la plus fermée du trapèze vocalique), alors qu'il signifiait au départ 'image peinte avec du vermillon':

(T)hus the agreement between sound and sense is of late growth in miniature, which now, on account of the $i$, has come to mean 'a small picture', while at first it meant 'image painted with minium or vermilion' (Jespersen, 1964: 408).

Notons également qu'Emile Benveniste, dans un article publié dans Acta linguisticaet repris dans le tome 1 des Problèmes de linguistique générale (1966: 49-55), avait montré qu'entre le signifié et le signifiant le lien ne saurait être arbitraire et qu'il était au contraire nécessaire, puisque l'un ne s'évoquait pas sans l'autre.

Enfin, nombreux sont ceux qui soutiennent l'idée de motivation phonétique aujourd'hui, et ce pour différentes langues. Les travaux de Didier Bottineau, par exemple, vont dans ce sens avec l'anglais, et ceux de Mihaï Dat et Georges Bohas avec les langues sémitiques. La mimophonie est également intégrée par Philippe Monneret à ses études sur le rôle de l'analogie dans le langage (voir par exemple Monneret, 2004).

Cette introduction était nécessaire pour rappeler que l'arbitraire du signe était loin d'avoir toujours été et d'être encore un principe incontesté. La théorie mimophonique a été soutenue à des degrés divers dans l'histoire de 
la linguistique, et ce pour des raisons idéologiques qu'il serait intéressant d'étudier. Nous ne pensons pas pour notre part que l'ensemble du langage soit motivé, mais nous pensons que les procédés mimophoniques y sont néanmoins présents. Nous voudrions montrer que les suffixes appréciatifs de l'espagnol, qui sont avant tout des morphèmes expressifs, sont également motivés phonétiquement.

Le triangle vocalique de l'espagnol présente comme voyelle la plus fermée le $i$ et comme voyelle la plus ouverte le $a$. Or, on sait, et il semble même que ce soit là un phénomène de grammaire générale, que le $i$ évoque psychologiquement tout ce qui est petit(voir par exemple l'article «Recherches sur le symbolisme phonétique» de Sapir, 1991), dans la mesure où son articulation requiert une aperture minimale, alors que le $a$ évoque au contraire ce qui est grand et demande un degré d'aperture élevé. Bien sûr, tout n'est ici qu'affaire de statistiques, mais toujours est-il que le lexique indo-européen présente davantage de termes en iqui évoquent l'idée de petitesse que de termes en $a$. Dans un cas comme la suffixation appréciative, qui nous intéresse ici, la motivation semble extrêmement élevée. Nous l'analyserons en deux temps, proposant deux principes d'explication distincts qui permettront de rendre compte à la fois des appréciatifs à valeur véritablement diminutive et augmentative, puis des appréciatifs à valeur affective et péjorative. Bien sûr, ce que nous observons ici n'est qu'une tendance générale, et l'on pourra trouver quelques contre-exemples dans des variantes dialectales de l'espagnol; mais cette tendance générale et majoritaire nous semble assez significative pour que nous puissions parler de mimophonie des suffixes appréciatifs de l'espagnol.

Il convient tout d'abord de bien souligner que, parmi les suffixes appréciatifs qui ont une valeur diminutive possible, et une valeur diminutive physique, les apertures sont faibles, en $i$ et en $u$ essentiellement. On ne trouve là aucun $a$. Ces suffixes diminutifs sont les suivants : ito, illo, ico, ete, uco, uelo, in, ino. Tous peuvent évoquer la diminution physique, et tous ont un degré d'aperture minimale, à l'exception de ete, dont le degré d'aperture est certes plus élevé. Ces suffixes peuvent bien sûr avoir de tout autres valeurs, affectives notamment, mais toujours est-il que leur possibilité d'exprimer la diminution concrète ou abstraite se reflète bien phonétiquement à travers la présence d'une voyelle d'aperture faible. La valeur affective est, en outre, liée à celle de petitesse, comme nous le rappellerons dans un deuxième temps en étudiant la mimophonie des appréciatifs subjectifs.

Mais restons un instant encore dans le domaine objectif et dans celui de l'augmentatif cette fois. L'augmentatif évoque, comme son nom l'indique, ce qui est grand, ce qui est imposant; il est donc tout à fait naturel de le retrouver avec une voyelle d'aperture élevée, le $a$, ou avec le $o$ ouvert. 
Nous pourrons citer les augmentatifs suivants : azo, ón et ote, qui peuvent certes avoir d'autres valeurs, mais qui ont une valeur physique augmentative qui justifie leur aperture maximale.

On voit ainsi que la mimophonie est très présente dans les cas où les suffixes appréciatifs renvoient clairement à une réalité concrète et mesurable.

Mais il est à noter que des suffixes comme ito, ico ou uelo ont des valeurs affectives certaines, et que des suffixes comme azo et ote peuvent avoir des valeurs péjoratives. Nous ajouterons donc également à cette liste tous les suffixes péjoratifs relevés par la Real Academia (2009: 661) : aco, aca (bicharraco, libraco), acho, acha (amigacha), ajo, aja (cintajo), astro, astra (poetastro), etc.

Il s'agit ainsi de voir en quoi l'idée péjorative, et notamment celle de mépris, peut être liée à l'idée augmentative phonétiquement (et, par conséquent, psychologiquement), alors que les suffixes diminutifs se chargent de valeurs affectives importantes. En d'autres termes, pourquoi le $i$ évoque-t-il généralement ce pour quoi l'on éprouve de l'affection, et le $a$ ce pour quoi l'on éprouve du dégoût?

Nous voudrions proposer quelques éléments d'explication là encore. L'idée d'affection, tout d'abord, est liée à celle de diminution, comme on l'a bien souvent remarqué, et ne pose pas a priori de problème en ce qui concerne son explication. En revanche, pourquoi l'idée de mépris est-elle rendue phonétiquement par des voyelles d'aperture maximale, $a$ essentiellement? C'est que, alors que l'affection veut que l'on rapproche l'objet chéri de soi, le mépris au contraire, et toute valeur péjorative en général, veut que l'on éloigne l'objet méprisé de soi, qu'on le rejette, qu'on marque une distance maximale vis-à-vis de lui. On voit que le apermet alors la distance la plus grande possible, puisque ce $a$ est la voyelle qui se trouve au sommet du triangle vocalique.

Un problème toutefois semble encore se poser, à savoir le fait que certains diminutifs peuvent avoir une valeur péjorative et certains augmentatifs au contraire une valeur méliorative. Mais c'est là un phénomène qui est d'une part loin d'être majoritaire statistiquement, et qui, d'autre part, peut trouver une explication dans un principe comme celui d'Inchaurralde tel qu'il a été modifié par SantibáñezSáenz (1999), qui écrit que dans la mesure où les entités petites peuvent sembler négligeables, elles ont parfois une valeur péjorative, alors que ce qui est grand peut au contraire paraître majestueux et donc être mélioratif.

Enfin, on peut également se demander dans quelle mesure les faits de mimophonie éclairent également les faits diachroniques. Si nous laissons de côté les suffixes appréciatifs à proprement parler et que nous étudions des 
oppositions de grandeur dans des paires comme cesto/cesta, canasto/canasta, cántaro/cántara, on voit que le féminin est plus grand que le masculin. W. von Wartburg, cité par Manuel Alvar et Bernard Pottier à qui nous reprenons ces exemples (1983: 36), nous donne une explication de type historique qui consiste à dire que les noms en $a$ qui ont une valeur augmentative viennent majoritairement d'anciens neutres en $a$ à valeur collective. C'est bien sûr là un élément d'explication; toutefois, on peut également se demander si des faits de mimophonie n'ont pas joué un rôle important, ici l'aperture élevée du $a$ pour exprimer ce qui est plus grand, le $o$, d'aperture moins élevée, exprimant ce qui est plus petit.

On a vu que l'augmentatif et le péjoratif étaient traduits phonétiquement par des voyelles très ouvertes en espagnol, alors que le diminutif et l'affectif renvoyaient à des voyelles fermées. Dans un cas, nous avons le reflet phonétique d'une réalité mesurable et la mise à distance d'un objet déprécié; dans l'autre, le reflet phonétique de la diminution et le désir de rapprochement d'un objet chéri. Ce phénomène semble se retrouver également dans d'autres langues romanes, et notamment en portugais. Il conviendrait bien entendu de faire ici une étude systématique, mais il nous semble en tout cas que les langues romanes vont à peu près dans le même sens que l'espagnol, même si en espagnol la mimophonie est particulièrement frappante.

C'est, à travers cela, une preuve de plus en faveur de la motivation phonétique de certains morphèmes qui apparaît, et une remise en question de plus du principe de l'arbitraire du signe.

\section{RÉFÉRENCES}

Alvar, Manuel et Bernard Pottier. 1983. Morfología histórica del español. Madrid: Editorial Gredos.

Court de Gébelin, Antoine. 1776. Histoire naturelle de la parole, ou Précis de l'origine du langage et de la grammaire universelle. Paris: L'auteur (livre numérisé par la BNF).

De Brosses, Charles. 1765. Traité de la formation mécanique des langues. Paris: Chez Saillant, Vincent, Desaint(livre numérisé par la BNF).

Huelmslev, Louis. 1968. Principes de grammaire générale. København:Munksgaard.

Jespersen, Отto. 1964. Language: its nature, development, and origin. London: George Allen.

Monneret, Philippe. 2004. Essais de linguistique analogique. Dijon: A.B.E.L.L.

Platon.1961. Cratyle, in Oeuvres complètes, tome 5. Texte établi par Louis Méridier. Paris: Les Belles Lettres.

Real Academia Española. 2009. Nueva gramática de la lengua española. Morfología, Sintaxis, tome 1. Madrid: Asociación de Academias de la Lengua Española. 
Santibáñez Sáenz, Francisco. 1999. Conceptual interaction and Spanish diminutives. Cuadernos de investigación filológica 25: 173-190.

SAPIR, EDWARD. 1991. Linguistique. Paris: Gallimard.

Saussure, Ferdinand de.1967. Cours de linguistique générale. Paris: Payot. 